\title{
SPIN ASYMMETRIES FOR EXCLUSIVE AND SEMI-EXCLUSIVE REACTIONS WITH CLAS
}

\author{
A. BISELLI \\ FOR THE CLAS COLLABORATION \\ Carnegie Mellon University, \\ 5000 Forbes Ave, \\ Pittsburgh, PA 15213, USA \\ E-mail: biselli@jlab.org
}

\begin{abstract}
An extensive experimental program to measure the spin structure of the nucleons is being carried out with the CLAS detector at Jefferson Lab using a longitudinally polarized electron beam incident on a longitudinally polarized target. Spin degrees of freedom offer new tools to explore the baryon structure and test the many theoretical approaches that attempt to characterize it, such as effective Lagrangian models and transverse momentum dependent parton distributions. I will present preliminary results for single and double spin asymmetries for exclusive $\pi^{0}, \pi^{+}$, and $\eta$ electroproduction in the resonance region compared with unitary isobar and dynamical models, as well as $\rho$ electroproduction for DIS kinematics. I will also report on an analysis of the semi-inclusive and exclusive channels where double and single spin asymmetries were used to study transverse momentum dependent parton distributions.
\end{abstract}

\section{Introduction}

In recent years the CLAS collaboration completed data taking for an experimental program dedicated to measuring inclusive, semi-exclusive, and exclusive reactions using longitudinally polarized electrons scattered off longitudinally polarized protons and deuterons. The experiments were conducted with the CLAS detector at Jefferson Lab. The running period for the experiment was split into two parts: the first part was completed in 1998 with a total of about $10^{9}$ triggers at two beam energies, 2.5 and $4 \mathrm{GeV}$, leading to publications for both inclusive ${ }^{1,2}$ and exclusive channels ${ }^{3,4}$. The second part of the run was conducted in 2000-2001 collecting a total of 2.3 $\times 10^{10}$ triggers at beam energies of $1.6,2.4,4.2$, and $5.7 \mathrm{GeV}$.

Here I report on the on-going analyses of the 1.6 and $5.7 \mathrm{GeV}$ data. In particular I will present preliminary results in the resonance region for the exclusive channels $\vec{e} \vec{p} \rightarrow e^{\prime} p \pi^{0}$ and $\vec{e} \vec{p} \rightarrow e^{\prime} p \eta$ and the results in the 
DIS region for the exclusive reaction $\vec{e} \vec{p} \rightarrow e^{\prime} p \rho$ and for the semi-exclusive channel $\vec{e} \vec{p} \rightarrow e^{\prime} \pi^{+} X$.

\section{The experiment}

The data for this analysis were taken with the CLAS detector system ${ }^{5}$ in Hall B at Jefferson Laboratory in Newport News, VA. Since the CLAS detector uses a toroidal magnetic field, which is zero along the beam axis, it is possible to insert a polarized target into the detector. The $\operatorname{target}^{6}$, consisting of solid ${ }^{15} \mathrm{NH}_{3}$, was polarized using dynamic nuclear polarization and was immersed in a $T=1 K{ }^{4} \mathrm{He}$ cooling bath. The holding field of $B=5 T$ had a very high uniformity of $\frac{d B}{B}=10^{-4}$. With this setup, target polarizations of $P_{t}=+79 \%$ and $P_{t}=-72 \%$ were achieved. In addition to the ${ }^{15} \mathrm{NH}_{3}$ target a solid ${ }^{12} \mathrm{C}$ target and an empty target cell were used for background studies.

\section{Exclusive channels}

The exclusive cross-section for meson electroproduction can be written as

$$
\frac{d \sigma}{d \Omega^{*}}=\frac{|\vec{k}|}{k_{\gamma}^{c m}}\left\{\frac{d \sigma_{0}}{d \Omega^{*}}+h \frac{d \sigma_{e}}{d \Omega^{*}}+P_{t} \frac{d \sigma_{t}}{d \Omega^{*}}+h P_{t} \frac{d \sigma_{e t}}{d \Omega^{*}}\right\}
$$

where $d \Omega^{*}=\sin \theta^{*} d \theta^{*} d \phi^{*}$ is the solid angle of the meson in the hadronic center of mass (c.m.), $\vec{k}$ is the momentum of the meson, $k_{\gamma}^{c m}$ is the real photon equivalent energy in the c.m. frame, $h$ is the electron helicity and $P_{t}$ is the target proton polarization. It is clear that by performing polarized beam and target experiments one can access the contributions to the cross section, $d \sigma_{e} / d \Omega^{*}, d \sigma_{t} / d \Omega^{*}$, and $d \sigma_{e t} / d \Omega^{*}$, in addition to the well known unpolarized cross section $d \sigma_{0} / d \Omega^{*}$, thus increasing our understanding of resonance production and background reactions. To isolate these terms, one can combine the data to extract the asymmetries $A_{t} \equiv \frac{\sigma_{t}}{d \Omega^{*}} / \frac{\sigma_{0}}{d \Omega^{*}}$ and $A_{e t} \equiv \frac{\sigma_{e t}}{d \Omega^{*}} / \frac{\sigma_{0}}{d \Omega^{*}}$, which have the experimental advantage of being nearly independent of acceptance and detector efficiency as compared to a cross section measurement. Given the number of counts per charge for events in the four possible combinations of beam $(i)$ and target $(j)$ polarizations, $N_{i j}$, the experimental definition of the asymmetries are:

$$
\begin{gathered}
A_{t}=\frac{1}{P_{t}^{b}} \frac{\left(N_{\uparrow \uparrow}+N_{\downarrow \uparrow}\right)-\left(N_{\uparrow \downarrow}+N_{\downarrow \downarrow}\right)}{\left(N_{\uparrow \uparrow}+N_{\downarrow \uparrow}\right)+\alpha\left(N_{\uparrow \downarrow}+N_{\downarrow \downarrow}\right)-2(1+\alpha) \frac{\sigma}{0}^{2} \Omega^{*}} \\
A_{e t}=\frac{1}{P_{e} P_{t}^{b}} \frac{-\left(N_{\uparrow \uparrow}-N_{\downarrow \uparrow}\right)+\left(N_{\uparrow \downarrow}-N_{\downarrow \downarrow}\right)}{\left(N_{\uparrow \uparrow}+N_{\downarrow \uparrow}\right)+\alpha\left(N_{\uparrow \downarrow}+N_{\downarrow \downarrow}\right)-2(1+\alpha) \frac{\sigma}{0}^{*} N},
\end{gathered}
$$


where $\frac{\sigma_{0}}{d \Omega^{*}}{ }^{N}$ is the contribution from the scattering off ${ }^{15} \mathrm{~N}$ nuclei and the liquid helium coolant, $P_{e}$ is the beam polarization, $P_{t}^{a}$ and $P_{t}^{b}$, are the magnitudes of positive and negative target polarizations, respectively, and

$$
\alpha \equiv \frac{P_{t}^{a}}{P_{t}^{b}} .
$$

The contribution of the ${ }^{15} \mathrm{~N}$ background was removed by using data from separate runs with a ${ }^{12} \mathrm{C}$ target. The products $P_{e} P_{t}^{a, b}$ were experimentally extracted using the well known asymmetry of the elastic reaction.

\section{1. $\pi^{0}$ electroproduction}

For the $\pi^{0}$ electroproduction analysis, the $1.6 \mathrm{GeV} \vec{p}\left(\vec{e}, e^{\prime}, p\right) \pi^{0}$ data were considered. The $\pi^{0}$ was identified with a missing mass cut. The target and double spin asymmetries as a function of the decay angles in the c.m. frame of the pion were extracted as in Eq. 2 in an invariant mass $(W)$ range from 1.1 to $1.6 \mathrm{GeV} / c^{2}$, and momentum transfer $\left(Q^{2}\right)$ range from 0.22 to $0.77 \mathrm{GeV}^{2} / c^{2}$. A sample of the results is shown in Fig 1 . The results were tested against the Mainz unitary isobar model MAID ${ }^{7}$ and the dynamical model $\mathrm{DMT}^{8}$ in the whole $W$ range. For $W$ up to $1.3 \mathrm{GeV} / c^{2}$ an additional comparison with the model by T. Sato and H. Lee ${ }^{9,10}$, which is a model specific for the $\Delta(1232)$ resonance, was performed. All these models are effective field theories that give predictions on the polarized observables with the free parameters associated with the model constrained by fitting unpolarized cross section data.

To quantitatively determine the agreement between the data and the model, a simultaneous $\chi^{2}$ comparison of all angular distributions for all $Q^{2}$ intervals was performed and the results are listed in Table 1 . The $\chi^{2}$ com-

Table 1. $\chi^{2}$ per number of degrees of freedom comparison between data and the three theoretical models.

\begin{tabular}{c|c|c|c|c}
\hline Model & $A_{t}$ & $A_{\text {et }}$ & $A_{t}$ & $A_{\text {et }}$ \\
\hline & \multicolumn{2}{|c|}{$\begin{array}{c}W<1.3 \mathrm{GeV} / c^{2} \\
\text { ndf }=1440\end{array}$} & \multicolumn{2}{c}{$\begin{array}{c}\text { }>1.3 \mathrm{GeV} / c^{2} \\
\mathrm{ndf}=1080\end{array}$} \\
& \multicolumn{2}{|c|}{} & \multicolumn{2}{|c}{} \\
\hline $\mathrm{MAID03}^{7}$ & 1.89 & 1.05 & 1.14 & 1.46 \\
$\mathrm{SL}^{9,10}$ & 1.02 & 1.09 & - & - \\
$\mathrm{DMT}^{8}$ & 2.27 & 1.04 & 1.61 & 1.02 \\
\hline
\end{tabular}

parison in the $\Delta(1232)$ region $\left(W<1.3 \mathrm{GeV} / c^{2}\right)$ shows overall agreement 
between the data and the model predictions of the double spin asymmetries $A_{e t}$, which are dominated by the $\left|M_{1+}\right|^{2}$ term. The models, however, differ in their predictions of the target asymmetries $A_{t}$, which are sensitive to interference of the $\Delta(1232)$ resonance with background multipoles such as $E_{0+}, S_{0+}$, and $S_{1-}$. The $\chi^{2}$ comparison also shows a preference for the Sato and Lee model in the $\Delta(1232)$ region. These results are consistent with the already published comparison in ref. ${ }^{3}$, but with much improved statistical accuracy. The $\chi^{2}$ comparison for invariant masses above the $\Delta(1232)$ resonance, where uncertainties in the models are bigger due to the many overlapping resonances, show discrepancies in both the target and double spin asymmetries, but further work is needed to understand the sensitivity of the asymmetries to the different contributions.

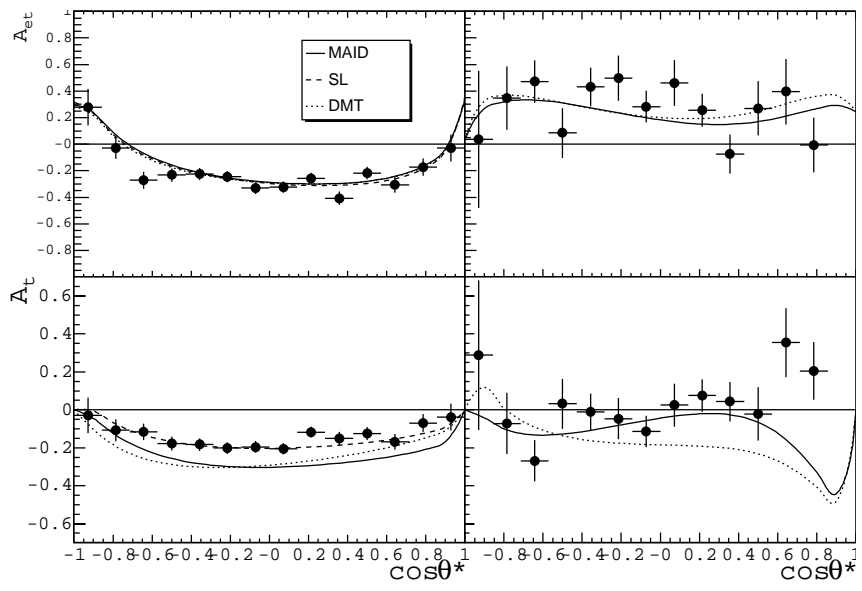

Figure 1. Asymmetry $A_{e t}$ (top) and $A_{t}$ (bottom) as a function of the c.m. polar angle of the pion, $\cos \theta^{*}$, for $-144.0^{\circ}<\phi^{*}<-108.0^{\circ}, 0.223<Q^{2}<0.379 \mathrm{GeV}^{2} / c^{2}$ and for the $1.20<W<1.25 \mathrm{GeV} / c^{2}$ (left) and $1.4<W<1.5 \mathrm{GeV} / c^{2}$ (right) intervals. The curves represent the predictions from the MAID2003 model (solid), DMT (dotted), and the Sato-Lee model (dashed).

\section{2. $\eta$ electroproduction}

For the $\eta$ electroproduction analysis, data with beam energies of $1.6 \mathrm{GeV}$ and $5.7 \mathrm{GeV}$ were considered. The $\eta$ was identified with a missing mass cut. The target and double spin asymmetries as a function of the decay angles in the c.m. frame of the $\eta$ were extracted. Assuming $S_{11}(1535)$ dominance 
and therefore dominance of the $E_{0+}$ and $L_{0+}$ multipoles, one can reduce the double spin asymmetry to first order to

$$
A_{e t} \sim\left(1-\epsilon \frac{E}{E^{\prime}}\right) \equiv D=\text { const },
$$

independent of $\theta^{*}$ and $\phi^{*}$, where $E$ is the beam energy, $E^{\prime}$ is the scattered electron energy, $\epsilon \equiv\left(1+2 \frac{|\overrightarrow{\mid}|^{2}}{Q^{2}} \tan ^{2} \frac{\theta_{e}}{2}\right)^{-1}, \vec{q}$ is the momentum transfer three vector, and $\theta_{e}$ is the electron scattering angle. This constant value $D$ corresponds to the depolarization of the virtual photon and $D \approx 0.75$ for $E=1.6 \mathrm{GeV}$ and $\approx 0.25$ for $E=5.7 \mathrm{GeV}$. The results for the $5.7 \mathrm{GeV}$ data in Fig. 2 show a constant behavior consistent with $S_{11}(1535)$ dominance. The target asymmetry results shown in Fig. 3 are very close to zero, indicating a strong $S_{11}(1535)$ resonance relative to the background. This is in contrast to the $\pi^{0}$ results that show a large target asymmetry in the $\Delta(1232)$ region. Both $A_{e t}$ and $A_{t}$ were compared to the Eta-MAID model ${ }^{11}$ which gives predictions in good agreement with the data.
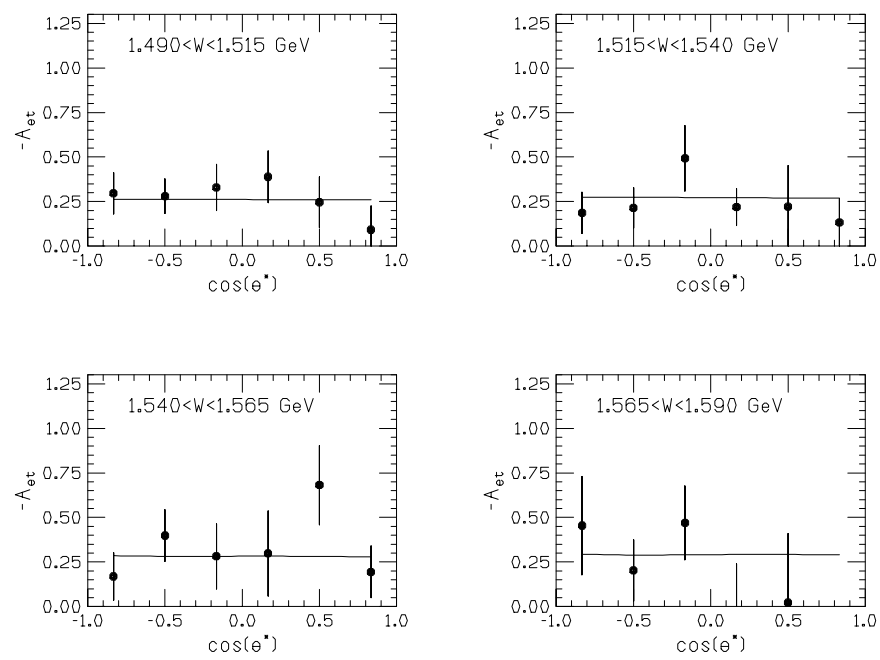

Figure 2. Asymmetry $A_{e t}$ as a function of the c.m. angle of the $\eta, \cos \theta^{*}$, for the 4 indicated $W$ intervals with a beam energy of $5.7 \mathrm{GeV}$ averaged over $\phi^{*}$. The curves represent the predictions of the Eta-MAID model.

\section{3. $\rho$ electroproduction}

The $\rho$ electroproduction analysis was performed in the DIS region, $W>1.8$ $\mathrm{GeV} / c^{2}$ and $Q^{2}>0.75 \mathrm{GeV}^{2} / c^{2}$, with the $5.7 \mathrm{GeV}$ data. To select a region 

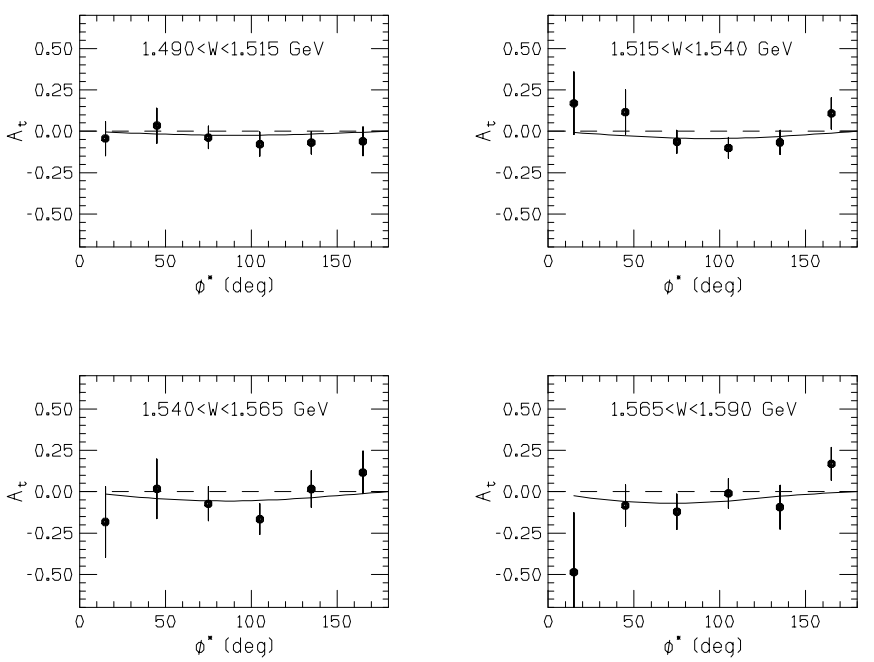

Figure 3. Asymmetry $A_{t}$ as a function of the c.m. azimuthal angle of the $\eta, \phi^{*}$, for the 4 indicated $W$ intervals with a beam energy of $1.6 \mathrm{GeV}$ averaged over $\cos \theta^{*}$. The curves represent the predictions of the Eta-MAID model.

dominated by $\rho$ production, the forward angle cut $t^{\prime}<0.4 \mathrm{GeV}^{2} / c^{2}$ was applied. The reaction of interest for selecting the $\rho$ is $\vec{e} \vec{p} \rightarrow e p \pi^{+} \pi^{-}$, but due to the limited acceptance, topologies with one missing particle were also considered. The $\rho$ was then identified with a missing mass cut on the $\pi^{+} \pi^{-}$ mass $\left(0.68 \mathrm{GeV} / c^{2}<M_{\pi \pi}<0.86 \mathrm{GeV} / c^{2}\right)$. The double spin asymmetry, $A_{e p}$, as a function of the invariant mass $W$ was extracted. By integrating over the $\phi^{*}$ angular distribution, $A_{e p}$ can be reduced to the form

$$
A_{e p}=D \frac{A_{1}+\eta A_{2}}{1+\epsilon R}
$$

with $\eta \equiv \tan \theta_{\gamma} \sqrt{2 \epsilon(1+\epsilon)}$, where $\theta_{\gamma}$ is the virtual photon angle with respect the beam direction and $R \equiv \sigma_{L} / \sigma_{T}$. Using the HERMES parameterization $^{12} R=0.35\left(Q^{2} / 0.59\right)^{0.62}$ and neglecting $A_{2}$, the asymmetry $A_{1}$ was calculated for different $Q^{2}$ values as shown in Fig. 4 . The results were compared to the Reggeon exchange model by Kochelev ${ }^{13}$ and the Generalized Vector Meson Dominance model by Fraas ${ }^{14}$. The data lie below the models, although the $W$ range might be too low for the models to be reliable. The asymmetry was also measured by HERMES at higher $Q^{2}$ and $W$ and was found consistent with the models, but still very close to zero within the error bars ${ }^{12}$. 


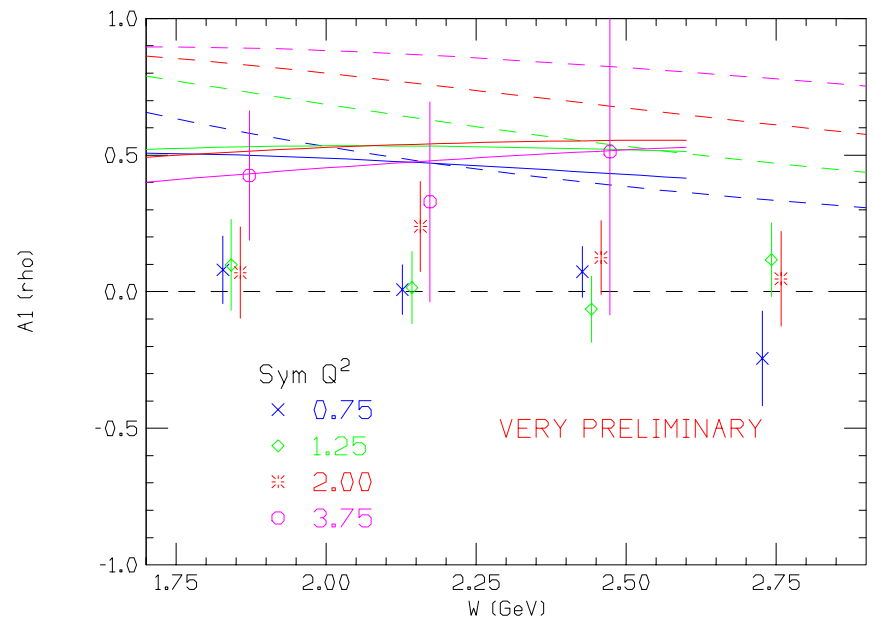

Figure 4. Asymmetry $A_{1}$ for $\vec{e} \vec{p} \rightarrow e p \rho$ as a function of $W$ averaged over $0<t^{\prime}<$ $0.4 \mathrm{GeV}^{2}$. The solid curves are from Kochelev and the dashed curves are from Fraas. The data points increase with $Q^{2}$ from left to right within each $W$ bin, and the dashed curves increase with $Q^{2}$ from bottom to top.

\section{Semi-exclusive $\pi^{+}$electroproduction}

The semi-exclusive analysis $\vec{e} \vec{p} \rightarrow e \pi^{+} X$ was performed using the $5.7 \mathrm{GeV}$ data, selecting the DIS region, where it is possible to interpret the results in terms of Generalized Parton Distributions and fragmentation functions ${ }^{15,16}$. In this framework one can factorize the cross section into distribution functions, which describe the quark scattering part, and fragmentation functions which describe the probability of a quark with a certain flavor to produce the final state hadron. The unpolarized cross section can be written as:

$$
\sigma_{U U} \propto\left(2-2 y+y^{2}\right) \sum_{q, \bar{q}} e_{q}^{2} f_{1}^{q}(x) D_{1}^{q}(z),
$$

where $x=Q^{2} / 2 M \nu, y=\nu / E$, and $z=E_{h} / \nu . \nu=E-E^{\prime}$ is the virtual photon energy, $E$ and $E^{\prime}$ are the initial and final electron energies, and $E_{h}$ is the final hadron energy. The sum $\sum_{q, \bar{q}}$ is over quark flavors, $y$ and $z$ are the fraction of electron energy carried by the virtual photon and the fraction of the virtual photon energy carried by the pion, respectively. The $f_{1}^{q}(x)$ and $D_{1}^{q}(z)$ are the unpolarized distribution and fragmentation functions. In a similar way the double polarization cross section can be written as:

$$
\sigma_{L L} \propto \lambda S_{L} y(2-y) \sum_{q, \bar{q}} e_{q}^{2} g_{1}^{q}(x) D_{1}^{q}(z) .
$$


Assuming $u$ quark dominance the double spin asymmetry can be simply written as:

$$
A_{e t} \sim D A_{1} \sim \frac{g_{1}^{u}(x)}{f_{1}^{u}(x)}
$$

Fig. 5(a) shows the double spin asymmetry calculated for different $z$ values and demonstrates, within statistical errors, consistency with the factorization. The results were also compared with the World inclusive data fit $A_{1} \propto x^{0.727}$ with good agreement. The data were also used to extract the target asymmetry. In the case of a polarized target, the cross section has the following additional terms:

$$
\begin{aligned}
\sigma_{U L}^{\sin 2 \phi} & \propto S_{L} 2(1-y) \sin 2 \phi \sum_{q, \bar{q}} e_{q}^{2} x h_{1 L}^{\perp q}(x) H_{1}^{\perp q}(z), \\
\sigma_{U T}^{\sin \phi} & \propto S_{T}(1-y) \sin \left(\phi+\phi_{S}\right) \sum_{q, \bar{q}} e_{q}^{2} x h_{1}^{q}(x) H_{1}^{\perp q}(z), \\
& +S_{T}\left(1-y+y^{2} / 2\right) \sin \left(\phi-\phi_{S}\right) \sum_{q, \bar{q}} e_{q}^{2} x f_{1 T}^{\perp q}(x) D_{1}^{q}(z), \\
\sigma_{U L}^{\sin \phi} & \propto S_{L} \sin \phi(2-y) \sqrt{1-y} \frac{M}{Q} \sum_{q, \bar{q}} e_{q}^{2} x^{2} h_{L}^{q}(x) H_{1}^{\perp q}(z),
\end{aligned}
$$

where $S_{L}$ and $S_{T}$ are the longitudinal and transverse components of the target polarization with respect to the direction of the virtual photon, $\phi$ is the azimuthal angle between the scattering plane and the production plane ${ }^{a}$, and $\phi_{S}$ is the azimuthal angle of the transverse spin in the scattering plane. The Collins fragmentation functions $H_{1}^{\perp q}(z)$ appear with a unique $\sin 2 \phi$ factor in the cross section, so a measurement of non-zero $\sin 2 \phi$ dependence would be a clear signature of the Collins mechanism ${ }^{17}$. To isolate the $\sin 2 \phi$ or $\sin \phi$ one can calculate the average

$$
A_{U L}^{W(\phi)}=\frac{\int \sigma_{U L}(\phi) W(\phi) d \phi}{\int \sigma(\phi) d \phi},
$$

where

$$
W(\phi)=\sin (\phi) \text { or } W(\phi)=\sin (2 \phi),
$$

\footnotetext{
aThe scattering plane is formed by the initial and final momenta of the electron, and the production plane by the transverse momentum of the observed hadron and the virtual photon.
} 
which experimentally translates into:

$$
A_{U L}^{W(\phi)}=\frac{2}{P^{ \pm} N^{ \pm}} \sum_{i=1}^{N^{ \pm}} W\left(\phi_{i}\right) .
$$

Results for the moments are shown in Fig. 5(b) and indicate the first observation of a non-zero $\sin 2 \phi$ moment. The results for the $\sin \phi$ moment show consistency with the previous measurement from the HERMES experiment ${ }^{18}$ at $27.5 \mathrm{GeV}$ beam energy, and therefore indicate that the observables do not depend on the beam energy.

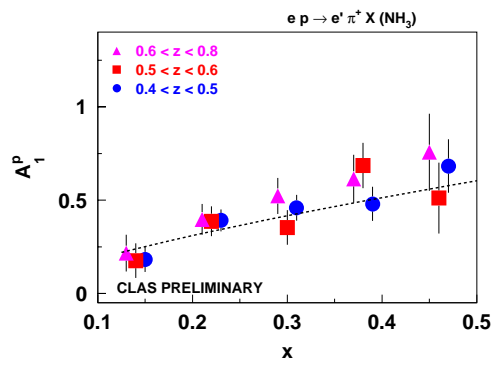

(a) $A_{1}$

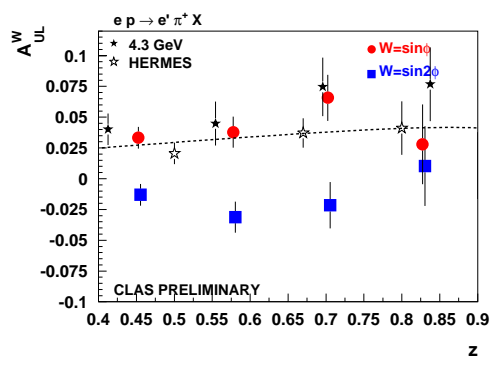

(b) $A_{U L}^{W(\phi)}$

Figure 5. (a) Double spin asymmetry as a function of $x$ for different $z$. (b) $A_{U L}^{\sin (\phi)}$ (circle) and $A_{U L}^{\sin (2 \phi)}$ (squares) as a function of $z$. Data are compared with $4.3 \mathrm{GeV}$ JLab data (filled star) and HERMES data at $27 \mathrm{GeV}$ (open stars)

\section{Outlook}

Target and double spin asymmetries for several exclusive and semi-exclusive channels in the resonance and the DIS regions were measured. High statistical samples allow us to extract asymmetries for several $W$ and $Q^{2}$ intervals to gain new knowledge about resonance and background contributions. The semi-exclusive channels also allow us to verify factorization and to investigate various terms introduced in the theoretical framework of the transverse momentum dependent parton distribution contributing to the cross section. 


\section{References}

1. R. Fatemi et al. [CLAS Collaboration], Phys. Rev. Lett. 91, 222002 (2003)

2. J. Yun et al. [CLAS Collaboration], Phys. Rev. C 67, 055204 (2003)

3. A. Biselli et al. [CLAS Collaboration], Phys. Rev. C 68, 035202 (2003)

4. R. De Vita et al. [CLAS Collaboration], Phys. Rev. Lett. 88, 082001 (2002)

5. B. A. Mecking et al. [CLAS Collaboration], Nucl. Instrum. Meth. A 503, 513 (2003).

6. C. D. Keith et al., Nucl. Instrum. Meth. A 501, 327 (2003).

7. D. Drechsel, O. Hanstein, S.S. Kamalov and L. Tiator, Nucl. Phys. A645, 145 (1999)

8. S. S. Kamalov, G. Y. Chen, S. N. Yang, D. Drechsel and L. Tiator, Lett. B 522, 27 (2001)

9. T. Sato and T.S. Lee, Phys. Rev. C54, 2660 (1996)

10. H. Lee, Nucl. Phys. A513 (1990) 511

11. W. T. Chiang, S. N. Yang, L. Tiator and D. Drechsel, Nucl. Phys. A 700, 429 (2002)

12. A. Airapetian et al. [HERMES Collaboration], Eur. Phys. J. C 29, 171 (2003)

13. N. I. Kochelev, D. P. Min, Y. s. Oh, V. Vento and A. V. Vinnikov, Phys. Rev. D 61, 094008 (2000)

14. H. Fraas, Nucl. Phys. B 113, 532 (1976).

15. P. J. Mulders and R. D. Tangerman, Nucl. Phys. B 461, 197 (1996)

16. D. Boer and P. J. Mulders, Phys. Rev. D 57, 5780 (1998)

17. J. C. Collins, Nucl. Phys. B 396, 161 (1993)

18. A. Airapetyan et al. [HERMES collaboration] , Phys.Rev.Lett. 84, 4047 (2000). 